Supplement of The Cryosphere, 14, 2115-2135, 2020

https://doi.org/10.5194/tc-14-2115-2020-supplement

(C) Author(s) 2020. This work is distributed under

the Creative Commons Attribution 4.0 License.

(c) (1)

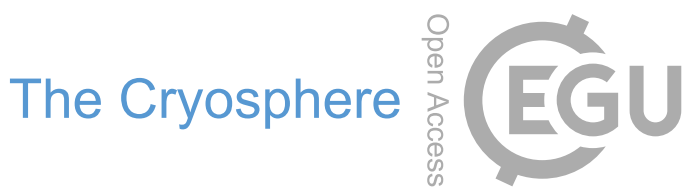

Supplement of

\title{
Past ice sheet-seabed interactions in the northeastern Weddell Sea embayment, Antarctica
}

Jan Erik Arndt et al.

Correspondence to: Jan Erik Arndt (jan.erik.arndt@awi.de)

The copyright of individual parts of the supplement might differ from the CC BY 4.0 License. 
Table S1: Coordinates, water depths and recoveries of gravity cores (GC) and piston cores (PC) deployed in the study area.

\begin{tabular}{|c|c|c|c|c|c|c|c|}
\hline Core ID & Cruise ID & $\begin{array}{c}\text { Corer } \\
\text { type }\end{array}$ & $\begin{array}{c}\text { Latitude } \\
\quad\left({ }^{\circ}\right)\end{array}$ & $\begin{array}{c}\text { Longitude } \\
\left({ }^{\circ}\right)\end{array}$ & $\begin{array}{c}\text { Water depth } \\
\text { (m) }\end{array}$ & $\begin{array}{c}\text { Recovery } \\
\text { (m) }\end{array}$ & Reference \\
\hline GC631 & JR244 & $\mathrm{GC}$ & -75.1238 & 26.6918 & -261 & 0 & this study \\
\hline GC632 & JR244 & $\mathrm{GC}$ & -75.1277 & -26.6920 & -256 & 0 & this study \\
\hline GC633 & JR244 & GC & -75.1055 & -26.6568 & -314 & 0 & this study \\
\hline GC634 & JR244 & GC & -75.0070 & -25.4493 & -500 & 0.145 & this study \\
\hline GC635 & JR244 & GC & -74.9917 & -25.4635 & -494 & 1.17 & this study \\
\hline GC636 & JR244 & GC & -74.8118 & -25.4755 & -626 & 1.77 & this study \\
\hline GC637 & JR244 & $\mathrm{GC}$ & -74.6838 & -25.5817 & -606 & 2.7 & this study \\
\hline 013 & IWSOE68 & GC & -75.4500 & -26.5500 & -507 & 0.9 & Anderson et al. (1981) \\
\hline $3-7-1$ & IWSOE70 & $\mathrm{PC}$ & -75.4170 & -26.4670 & -235 & 5.3 & Anderson et al. (1981) \\
\hline $3-10-1$ & IWSOE70 & $\mathrm{PC}$ & -74.9000 & -25.9000 & -490 & 0.06 & Anderson et al. (1981) \\
\hline $3-11-1$ & IWSOE70 & $\mathrm{PC}$ & -74.4500 & -25.7170 & -528 & 0.06 & Anderson et al. (1981) \\
\hline PS1637-3 & ANT-VI/3 & $\mathrm{GC}$ & -74.7630 & -26.4420 & -445 & 0.6 & $\begin{array}{c}\text { Grobe and Fütterer } \\
\text { (2015) }\end{array}$ \\
\hline PS111_139-1 & PS111 & GC & -74.8251 & -25.2659 & -669 & 0 & Schröder (2018) \\
\hline PS111_140-2 & PS111 & GC & -75.1325 & -26.6295 & -341 & 0 & Schröder (2018) \\
\hline PS111_142-1 & PS111 & $\mathrm{GC}$ & -75.2169 & -27.4585 & -373 & 0.2 & Schröder (2018) \\
\hline
\end{tabular}




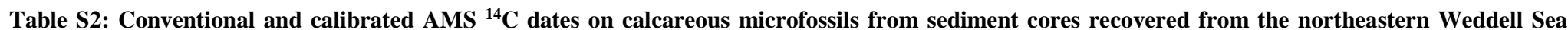

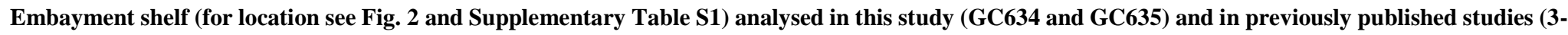
7-1, Stolldorf et al., 2012; Anderson et al., 1980; Anderson et al., 1981).

\begin{tabular}{|c|c|c|c|c|c|c|c|c|c|}
\hline \multirow[t]{2}{*}{ Lab. sample ID } & \multirow[t]{2}{*}{ Core ID } & \multirow{2}{*}{$\begin{array}{l}\text { Corer } \\
\text { type }^{\#}\end{array}$} & \multirow{2}{*}{$\begin{array}{c}\text { Sample } \\
\text { depth } \\
(\mathrm{cm})\end{array}$} & \multirow{2}{*}{$\begin{array}{c}\text { Mat. } \\
\text { dated* }\end{array}$} & \multirow{2}{*}{$\begin{array}{l}{ }^{14} \mathrm{C} \text { age } \\
\text { (yrs BP) }\end{array}$} & \multirow{2}{*}{$\begin{array}{c} \pm 1 \sigma \\
(\operatorname{yrs} B P)\end{array}$} & \multicolumn{3}{|c|}{ Calibrated age $\pm 2 \sigma$} \\
\hline & & & & & & & $\begin{array}{c}\text { Min. age } \\
\text { (cal ka BP) }\end{array}$ & $\begin{array}{c}\text { Max. age } \\
\text { (cal ka BP) }\end{array}$ & $\begin{array}{c}\text { Rounded mean age } \\
\text { (cal ka BP) }\end{array}$ \\
\hline AA-27756 & $3-7-1$ & $\mathrm{PC}$ & 200 & $\mathrm{bF}$ & 26660 & 490 & 28589 & 30723 & 29660 \\
\hline CCAMS-95860 & $3-7-1$ & $\mathrm{PC}$ & $223-227$ & $\mathrm{bF}$ & 28930 & 160 & 31157 & 31802 & 31480 \\
\hline CCAMS-96250 & $3-7-1$ & $\mathrm{PC}$ & $223-227$ & $\mathrm{bF}$ & 29490 & 220 & 31500 & 32839 & 32170 \\
\hline CCAMS-95861 & $3-7-1$ & $\mathrm{PC}$ & $304-308$ & $\mathrm{bF}$ & 17980 & 50 & 20014 & 20430 & 20220 \\
\hline CCAMS-96165 & $3-7-1$ & $\mathrm{PC}$ & $304-308$ & $\mathrm{bF}$ & 13315 & 30 & 13842 & 14119 & 13980 \\
\hline CCAMS-96251 & $3-7-1$ & $\mathrm{PC}$ & $304-308$ & $\mathrm{bF}$ & 15330 & 45 & 16956 & 17410 & 17180 \\
\hline CCAMS-96262 & $3-7-1$ & $\mathrm{PC}$ & $304-308$ & $\mathrm{bF}$ & 14655 & 40 & 15966 & 16348 & 16160 \\
\hline CCAMS-96252 & $3-7-1$ & $\mathrm{PC}$ & $348-352$ & $\mathrm{bF}$ & 19400 & 70 & 21819 & 22289 & 22050 \\
\hline AA-27757 & $3-7-1$ & $\mathrm{PC}$ & 400 & $\mathrm{bF}$ & 13640 & 130 & 14087 & 15093 & 14590 \\
\hline ETH-69711 & GC634 & GC & 12 & $\mathrm{pF}$ & 5470 & 70 & 4651 & 5166 & 4910 \\
\hline ETH-69710 & GC634 & GC & 12 & $\mathrm{bF}$ & 5490 & 70 & 4717 & 5200 & 4960 \\
\hline ETH-69709 & GC635 & GC & 50 & B & 10475 & 90 & 10259 & 10783 & 10520 \\
\hline
\end{tabular}

\#Corer types: GC: gravity corer, PC: piston corer

*Material dated: B: bivalve shell, E: echinoid spine, bF: benthic foraminifera, $\mathrm{pF}$ : planktic foraminifera 


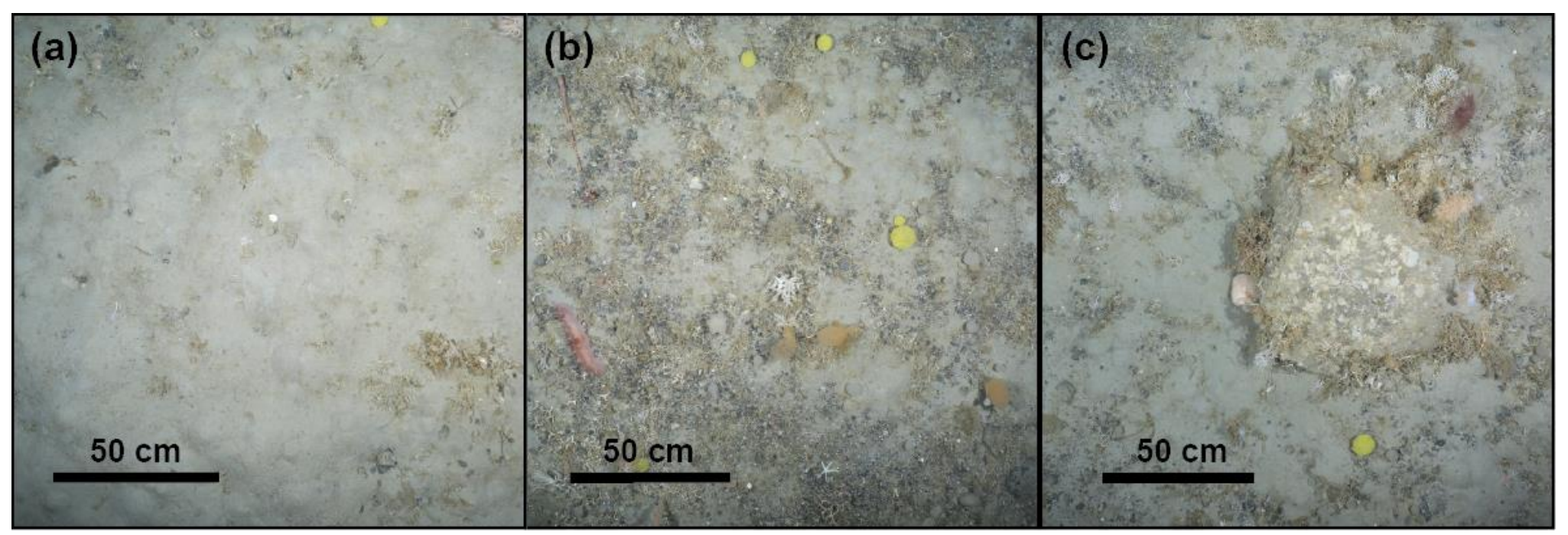

Figure S1: Ocean Floor Observation System (OFOS) seafloor images taken during RV Polarstern expedition PS96 on station 10-3 (Piepenburg, 2016) showing (a) a thin veneer of fine-grained mud, locally penetrated by hardrock pebbles and cobbles. Where the mud veneer is absent, the images show (b) a layer of hardrock pebbles and cobbles, and (c) locally, large cobbles and boulders, whose presence may explain the poor recovery of gravity cores in the area of Class $\mathrm{H}$ ramps. For location of photos see Fig. 2.

\section{References}

Anderson, J. B., Kurtz, D. D., Domack, E. W., and Balshaw, K. M.: Glacial and Glacial Marine Sediments of the Antarctic Continental Shelf, The Journal of Geology, 88, 399-414, 10.1086/628524, 1980.

Anderson, J. B., Davis, S. B., Domack, E., Kurtz, D. D., Balshaw, K. M., and Wright, R.: Marine Sediment Core Descriptions: IWSOE 68, 69, 70, Deep Freeze 79, Department of Geology, Rice University, Houston, USA, 1981.

Grobe, H. and Fütterer, D. K.: Documentation of sediment core PS1637-3, PANGAEA, 10.1594/PANGAEA.843865, 2015.

Piepenburg, D.: Seabed photographs taken along OFOS profiles during POLARSTERN cruise PS96 (ANT-XXXI/2 FROSN), PANGAEA, 10.1594/PANGAEA.862097, 2016.

Schröder, M.: The Expedition PS111 of the Research POLARSTERN to the southern Weddell Sea in 2018, Reports on polar and marine research, 718, Alfred Wegener Institute for Polar and Marine Research, Bremerhaven, Germany, 10.2312/BzPM_0718_2018, 2018.

Stolldorf, T., Schenke, H.-W., and Anderson, J. B.: LGM ice sheet extent in the Weddell Sea: evidence for diachronous behavior of Antarctic Ice Sheets, Quat. Sci. Rev., 48, 20-31, 10.1016/j.quascirev.2012.05.017, 2012. 